\title{
White matter damage due to vascular, tau, and TDP-43 pathologies and its relevance to cognition
}

\author{
Sheelakumari Raghavan', Scott A. Przybelski ${ }^{2}$, Robert I. Reid ${ }^{3}$, Timothy G. Lesnick ${ }^{2}$, Vijay K. Ramanan, \\ Hugo Botha ${ }^{4}$, Billie J. Matchett ${ }^{5}$, Melissa E. Murray ${ }^{5}$, R. Ross Reichard ${ }^{6}$, David S. Knopmann, \\ Jonathan Graff-Radford ${ }^{4}$, David T. Jones ${ }^{4}$, Val J. Lowe ${ }^{1}$, Michelle M. Mielke ${ }^{2,4}$, Mary M. Machulda ${ }^{7}$, \\ Ronald C. Petersen ${ }^{4}$, Kejal Kantarci ${ }^{1}$, Jennifer L. Whitwell ${ }^{1}$, Keith A. Josephs ${ }^{4}$, Clifford R. Jack Jr ${ }^{1}$ and \\ Prashanthi Vemuri ${ }^{* *}$ (1)
}

\begin{abstract}
Multi-compartment modelling of white matter microstructure using Neurite Orientation Dispersion and Density Imaging (NODDI) can provide information on white matter health through neurite density index and free water measures. We hypothesized that cerebrovascular disease, Alzheimer's disease, and TDP-43 proteinopathy would be associated with distinct NODDI readouts of white matter damage which would be informative for identifying the substrate for cognitive impairment. We identified two independent cohorts with multi-shell diffusion MRI, amyloid and tau PET, and cognitive assessments: specifically, a population-based cohort of 347 elderly randomly sampled from the Olmsted county, Minnesota, population and a clinical research-based cohort of 61 amyloid positive Alzheimer's dementia participants. We observed an increase in free water and decrease in neurite density using NODDI measures in the genu of the corpus callosum associated with vascular risk factors, which we refer to as the vascular white matter component. Tau PET signal reflective of 3R/4R tau deposition was associated with worsening neurite density index in the temporal white matter where we measured parahippocampal cingulum and inferior temporal white matter bundles. Worsening temporal white matter neurite density was associated with (antemortem confirmed) FDG TDP-43 signature. Post-mortem neuropathologic data on a small subset of this sample lend support to our findings. In the community-dwelling cohort where vascular disease was more prevalent, the NODDI vascular white matter component explained variability in global cognition (partial $R^{2}$ of free water and neurite density $=8.3 \%$ ) and MMSE performance (8.2\%) which was comparable to amyloid PET (7.4\% for global cognition and $6.6 \%$ for memory). In the AD dementia cohort, tau deposition was the greatest contributor to cognitive performance (9.6\%), but there was also a non-trivial contribution of the temporal white matter component (8.5\%) to cognitive performance. The differences observed between the two cohorts were reflective of their distinct clinical composition. White matter microstructural damage assessed using advanced diffusion models may add significant value for distinguishing the underlying substrate (whether cerebrovascular disease versus neurodegenerative disease caused by tau deposition or TDP-43 pathology) for cognitive impairment in older adults.
\end{abstract}

*Correspondence: vemuri.prashanthi@mayo.edu

${ }^{1}$ Department of Radiology, Mayo Clinic, 200 First Street SW, Rochester, MN 55905, USA

Full list of author information is available at the end of the article permits use, sharing, adaptation, distribution and reproduction in any medium or format, as long as you give appropriate credit to the original author(s) and the source, provide a link to the Creative Commons licence, and indicate if changes were made. The images or other third party material in this article are included in the article's Creative Commons licence, unless indicated otherwise in a credit line to the material. If material is not included in the article's Creative Commons licence and your intended use is not permitted by statutory regulation or exceeds the permitted use, you will need to obtain permission directly from the copyright holder. To view a copy of this licence, visit http://creativecommons.org/licenses/by/4.0/. The Creative Commons Public Domain Dedication waiver (http://creativeco mmons.org/publicdomain/zero/1.0/) applies to the data made available in this article, unless otherwise stated in a credit line to the data. 
Keywords: Diffusion tensor imaging, Neurite dispersion density imaging, Cerebrovascular disease, Tau positron emission tomography, TAR DNA binding protein of $43 \mathrm{kDa}$

\section{Graphical abstract}

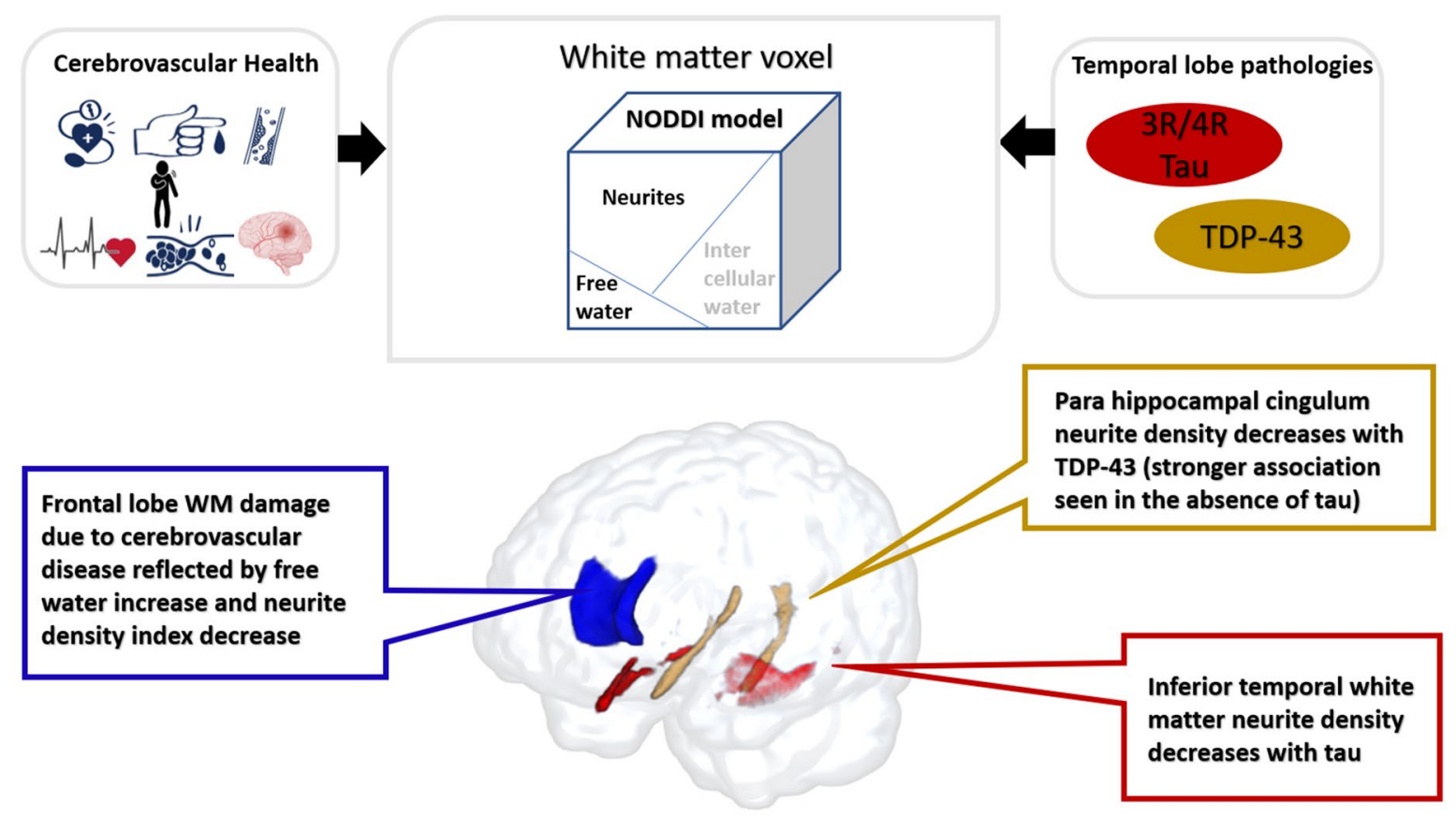

- Cerebrovascular disease, tau and TDP-43 proteinopathy cause distinct white matter microstructural damage measurable using advanced diffusion models.

- NODDI, acquired in a single setting of MRI, adds value by assessing the possible etiology and would inform on the substrate of cognitive impairment.

\section{Introduction}

The prevalence of neurodegenerative pathologies and cerebrovascular disease (CVD) increases with age [1]. Although Alzheimer's disease (AD) and CVD are the major contributors of cognitive decline in the elderly and are widely studied, evidence suggests the co-occurrence of medial temporal lobe (MTL) changes are caused by the TAR DNA binding protein of $43 \mathrm{kDa}$ (TDP-43) [24], also recently referred to as limbic-predominant agerelated TDP-43 encephalopathy (LATE) [5]. Typically, gray matter changes due to these age-related pathologies are the focus of most aging and dementia imaging studies. However, white matter (WM) damage associated with these pathological changes and their relevance to cognitive performance are not widely considered except for visible WM damage such as white matter hyperintensities.

Systemic vascular health and CVD have widespread impact on WM health [6, 7]. Greater damage has been observed in the frontal lobes related to anterior-posterior gradient in aging [8] and in CVD [9]. Our studies have also shown that frontal fibers are more vulnerable to age-related and CVD related changes $[6,10]$ and that the measurement of diffusion properties in the genu of the corpus callosum (we herein refer to this tract as "Genu") captures WM damage primarily characterized by aging and CVD. On the other hand, pathological hallmark features of AD accumulation of two proteins, extracellular amyloid- $\beta(A \beta)$ and intracellular neurofibrillary tangles composed of hyperphosphorylated tau [11] have a differential impact on WM health. $A \beta$ accumulation has minimal effects on WM health $[12,13]$. In contrast, temporal WM tracts may not only aid in the spreading of tau [14] but are progressively damaged because of increasing local tau toxicity [15]. Two tracts that are of particular interest in the context of tau are the parahippocampal cingulum bundle (commonly referred to as CGH - cingulum adjoining the hippocampus) [14] and inferior temporal WM (ITWM) [16]. The CGH WM tract is the primary 
connection with the hippocampus which is one of the earliest regions of tau deposition. The inferior temporal lobe tau which is affected in Braak stages III-IV; has high tau burden along the AD continuum [17].

The increasing accumulation of TDP-43 in the temporal lobes may also be hypothesized to cause damage to both CGH and ITWM tracts. While the gold standard for TDP-43 is an autopsy, recent evidence suggested [18F]fluorodeoxyglucose PET (FDG-PET) derived inferior/ medial temporal (IMT) hypometabolism as a diagnostic marker of TDP-43 proteinopathy associated hippocampal sclerosis aging [18]. A more recent study reported higher sensitivity and specificity of IMT to frontal supra orbital ratio (IMT/FSO) for predicting earlier stages of AD-related TDP-43(+) status [19]. One could hypothesize that the TDP-43 proteinopathy damages white matter in the temporal lobes in addition to neuronal dysfunction observed on FDG-PET.

Traditionally, diffusion tensor imaging (DTI) has been used extensively to study WM changes due to AD and CVD and their association with cognition [20,21]. However, the DTI findings are generally limited by their inability to accurately capture the nature of underlying tissue properties and their overestimation of voxels within crossing fibers [22, 23]. The recently developed Neurite and Orientation Dispersion Density Imaging (NODDI) model is a biophysically inspired method that provides a much more appropriate model for the estimation of biological measures using diffusion images [24] and enhances DTI findings by separating out free water contribution from the microstructural tissue compartment. NODDI model can estimate the Neurite Density Index (NDI, nominally the proxy for axonal density), Orientation Dispersion Index (ODI, an indicator of the lack of axonal alignment) and Isotropic Volume Fraction (ISOVF, the fraction of water diffusing freely without encountering membranes). To date, there is evidence that NODDI can be used to identify WM changes in aging $[25,26]$, neurodegenerative diseases [27-29], and associated cognitive deficits $[29,30]$. These NODDI measures also can provide information about neurite morphology and have shown sensitivity to tau [16]. Evidence from rodent's tau model showed reduced NDI in WM and hippocampus [28]. Additionally, reduced cortical NDI in temporal and frontal lobes have been shown [31, 32], and reduced NDI in the cortex was associated with worse cognitive performance in $\mathrm{AD}[29,31,32]$. We reported recently that NODDI derived indices (NDI and ISOVF) are sensitive to age and disease related WM damage and cognition [33].

Building on our previous work, in this paper we leveraged NODDI (NDI and ISOVF) measurements for evaluating aging and pathology related WM changes in the brain. Our central hypothesis was that NODDI measures from three WM tracts (Genu, ITWM, and CGH) would aid in differentially capturing WM damage due to CVD, tau, and TDP-43 pathologies and explaining variance in cognition in both the population-based cohort of Mayo Clinic Study of Aging (MCSA) and among clinically diagnosed $\mathrm{AD}$ dementia cases enrolled in the Mayo Alzheimer's Disease Research Center (ADRC). To test our hypothesis, we conducted two sets of analyses. First, we evaluated the association of vascular health, AD and TDP-43 biomarkers, and NODDI measurements in these three tracts. Second, we measured and compared the contribution of these NODDI measures for cognitive decline and compared this to amyloid and tau PET in the MCSA and ADRC cohorts.

\section{Materials and methods}

\section{Selection of participants}

Study participants were selected from the MCSA and ADRC. The selection criteria were the availability of multi-shell diffusion MRI (NODDI), amyloid and tau PET scans, vascular health indicators, and complete clinical assessments. FDG-PET was available in a subset of participants.

\section{MCSA participants}

The MCSA is an epidemiological cohort designed to investigate the prevalence, incidence, and risk factors for mild cognitive impairment (MCI) and dementia among the residents of Olmsted County, Minnesota [34]. The Olmsted county population was enumerated using Rochester Epidemiology Project (REP) medical records linkage system infrastructure $[35,36]$ and participants were randomly invited to participate in the MCSA using an ageand sex-stratified sampling frame. The clinical diagnosis of the participants was ascertained at the time of MRI using previously published criteria [37]. Based on the selection criteria, we included 347 participants from the MCSA ( $>60$ years) with the following clinical characteristics: 281 cognitively unimpaired, $61 \mathrm{MCI}$, and 5 dementia participants. Because all MCSA participants were recruited from the REP, we were able to include nurse abstracted vascular health indicators from the medical records-hypertension, diabetes mellitus, dyslipidemia, cardiac arrhythmias, coronary artery disease, congestive heart failure, and stroke to compute the composite score (CMC) as published previously [38].

\section{ADRC participants}

We included 61 amyloid positive clinically diagnosed AD dementia participants confirmed through consensus criteria from the Mayo Clinic-Rochester ADRC who were aged $\geq 65$ years to capture typical AD. 


\section{Standard protocol approvals, registrations, and patient consents}

The study was approved by the Mayo Clinic and Olmsted Medical Center institutional review boards and written informed consent was obtained from all participants or their qualified caregivers.

\section{Imaging}

\section{MRI acquisition and processing}

All MRIs were acquired on $3 \mathrm{~T}$ Siemens Prisma scanners using 64-channel receiver head coils. The acquisition protocols and analysis have been published previously for the MCSA [33]. All structural scans were acquired using magnetization prepared rapid gradient echo (MPRAGE) sequence with following parameters: $\mathrm{TR}=2300 \mathrm{~ms}$, $\mathrm{TE}=3.14 \mathrm{~ms}, \mathrm{TI}=945 \mathrm{~ms}$, flip angle $=9^{\circ}$, and isotropic resolution $=0.8 \mathrm{~mm}$. The diffusion scans were obtained with multi-band sequence with following parameters: $\mathrm{TR}=3400 \mathrm{~ms}, \mathrm{TE}=71 \mathrm{~ms}$, field of view $=232 \mathrm{~mm}$, and voxel size $=2.0 \mathrm{~mm}$ isotropic. The diffusion data consisted of 127 volumes with 13 non-diffusion-weighted images $\left(b=0 \mathrm{~s} / \mathrm{mm}^{2}\right)$, and 114 diffusion-encoding gradient directions $(6 \mathrm{~b}=500,48 \mathrm{~b}=1000$, and $60 \mathrm{~b}=2000 \mathrm{~s} /$ $\mathrm{mm}^{2}$ ). The data were evenly spread over the entire spherical shells using an electrostatic repulsion model [39]. Then, the diffusion images were processed as previously described (Raghavan et al. 2021). Briefly, the diffusionweighted images were denoised [40], corrected for head motion, eddy current distortion [41], and Gibbs ringing [42], then debiased [43]. The diffusion tensors were then fit using a nonlinear least squares fitting algorithm implemented in dipy [44]. NDI, and ISOVF maps were estimated using the Accelerated Microstructure Imaging via Convex Optimization (AMICO) implementation [45] of NODDI in Python. Our recent work showed a more consistent relationship of aging pathologies and cognition with NDI and ISOVF but not ODI [33] therefore we limited the analyses to NDI and ISOVF in this work. Median values of NODDI measures in the Genu, CGH, and ITWM were obtained by non-linearly registering the JHU "Eve" WM atlas [46] to each participant. The registration was calculated by aligning the "Eve" FA image to the participant FA using ANTS [47].

\section{Amyloid, tau, and FDG PET markers}

The acquisition, processing, and calculations of amyloid, tau, and FDG PET standardized uptake value ratio (SUVR) was described previously [18, 48]. Global amyloid SUVR for each participant was computed by calculating the median uptake over voxels in the prefrontal, orbitofrontal, parietal, temporal, anterior cingulate, and posterior cingulate/precuneus regions normalized by the median amyloid PET uptake in the cerebellar crus grey matter. Global tau SUVR for each participant was computed by calculating median tau PET uptake in the entorhinal, amygdala, parahippocampal, fusiform, inferior temporal, and middle temporal regions normalized by the median tau PET uptake in the cerebellar crus grey matter. We used continuous amyloid PET and tau PET as our primary AD biomarkers. We also utilized amyloid positivity (amyloid cutoff for positivity was SUVR $\geq 1.48$ ) and tau positivity (tau cutoff for positivity was SUVR $\geq 1.25$ ) established previously [48] for additional analyses.

For FDG-PET, we selected 3 regions of interest (ROI) from the ADIR122 atlas (available as part of the Mayo Clinic Adult Lifespan Template, nitrc.org/projects/ mcalt/) which have been associated with TDP-43 positivity: inferior temporal (IT), medial temporal (MT) (amygdala and hippocampus), and frontal supraorbital (FSO). Regional FDG SUVR for each participant was computed by calculating the median uptake from gray matter voxels in each ROI normalized by the median FDG uptake in the pons. Then, we assessed the ratio of IT to MT (IMT) and then IMT to FSO (IMT/FSO) and used as our FDG TDP-43 signature as this was proposed previously as a biomarker of TDP-43(+) status [19].

\section{Neuropathologic analysis}

Autopsy analysis was conducted in 9 deceased participants using a standardized dissection and sampling procedure recommended by Consortium to Establish a Registry for Alzheimer's Disease (CERAD) [49]. Formalin-fixed and paraffin-embedded left hemisphere brain sections were evaluated and were used for immunohistochemical analysis of TDP-43 (pS409/410, 1:10,000, Cosmo Bio). Cases were considered TDP-43(+) if TDP43 immunoreactive neuronal cytoplasmic inclusions, perivascular bi-lobed inclusions, or neuronal intranuclear inclusions were observed in the amygdala $[50,51]$.

\section{Cognitive performance}

The MCSA neuropsychological battery consists of nine tests covering 4 cognitive domains, as previously published [34, 37]. The primary outcome for the analysis was a global cognitive $\mathrm{z}$ score that was estimated from the $\mathrm{z}$-transformation of the average of the 4 cognitive domain $\mathrm{z}$ scores (memory, language, attention/executive, and visuo-spatial function) [52]. Across MCSA and ADRC studies, the common test was Mini-Mental State Examination (MMSE) [53], estimated from Short test of Mental Status [54], which we evaluated as an outcome for comparison. 


\section{Statistical analyses}

The participant characteristics were summarized using mean (standard deviation, SD) for the continuous variables and count (\%) for the categorical variables. Amyloid and tau distribution were skewed and hence analyzed with a log transformation.

Pearson partial correlations were used to identify the associations between vascular risk (CMC), AD biomarkers, FDG-TDP-43 signature with our tracts of interests after adjusting for age and sex.

Then, we fit separate multiple linear regression models with cognitive scores as an outcome variable (global cognition and MMSE in MCSA and MMSE in ADRC), with amyloid, tau, and regional diffusion measures as predictors, and adjusting for age, sex, education, and number of clinical visits (number of times a participant had previously completed cognitive testing in the study to account for practice effects). In addition, we fit multiple linear regression models using a combination of least absolute shrinkage and selection operator (LASSO) with alpha $=1$ and step-wise approaches to assess the added benefit of WM vascular component (Genu NDI and Genu ISOVF) and temporal WM diffusion metrics (CGH NDI and ITWM NDI) to AD biomarkers (amyloid and tau) for predicting cognitive performance. These models were also adjusted for age, sex, education, and number of clinical visits. We did not perform multiple comparison testing (which would rely on universal null hypothesis) because we did not want to inflate the probability of Type II error $[55,56]$.

\section{Results}

The characteristics of MCSA and ADRC data sets are shown in Table 1 . There were more males and APOE $\varepsilon 4$ carriers in the ADRC than the MCSA. All participants in the ADRC were amyloid positive with $92 \%$ of them were tau positive. Whereas, in the MCSA, $47 \%$ were amyloid positive with $36 \%$ of them were tau positive.

\section{NODDI measurements were associated with vascular risk factors, amyloid, tau, and TDP-43}

As explained in the introduction, the present study confirmed the relevance of Genu WM changes to cerebrovascular health. Both Genu ISOVF $(r=0.15$, $P=0.005)$ and Genu NDI $(\mathrm{r}=-0.15, P=0.005)$ were correlated with vascular risk measure i.e. CMC. A plot of the Genu NODDI measures with CMC in MCSA are shown in the first panel of Fig. 1. Therefore, we used both Genu ISOVF and Genu NDI as the components of CVD (WM vascular component). Amyloidosis was associated with an increase in Genu ISOVF $(r=0.13$,
Table 1 Characteristics table of subjects with the mean (SD) listed for the continuous variables and count (\%) for the categorical variables

\begin{tabular}{|c|c|c|}
\hline Characteristics & $\begin{array}{l}\text { MCSA } \\
n=347\end{array}$ & $\begin{array}{l}\text { ADRC } \\
n=61\end{array}$ \\
\hline \multicolumn{3}{|l|}{ Demographics and cognition } \\
\hline Age, yrs & $74.3(8.5)$ & $74.4(6.0)$ \\
\hline Males, no. (\%) & $185(53 \%)$ & $36(59 \%)$ \\
\hline APOE4, no. (\%) & $101(31 \%)$ & $32(71 \%)$ \\
\hline Education, yrs & $15.0(2.6)$ & $16.0(2.7)$ \\
\hline MMSE & $28.3(1.8)$ & $21.9(5.0)$ \\
\hline zGlobal & $-0.02(1.41)$ & NA \\
\hline CDR & $0.2(0.7)$ & $4.1(2.9)$ \\
\hline Cognitively Impaired, no. (\%) & $66(19 \%)$ & $61(100 \%)$ \\
\hline \multicolumn{3}{|l|}{ CVD markers } \\
\hline WMH & $1.03(1.04)$ & $1.35(1.19)$ \\
\hline Hypertension, no. (\%) & $221(64 \%)$ & $27(44 \%)$ \\
\hline Diabetes, no. (\%) & $56(16 \%)$ & $5(8 \%)$ \\
\hline Dyslipidemia, no. (\%) & $281(81 \%)$ & $21(34 \%)$ \\
\hline \multicolumn{3}{|l|}{$A D$ and TDP-43 markers } \\
\hline PIB SUVr & $1.67(0.48)$ & $2.43(0.44)$ \\
\hline Tau SUVr & $1.23(0.14)$ & $1.78(0.46)$ \\
\hline FDG TDP-43 $3^{\mathrm{a}}$ & $0.83(0.06)$ & $0.87(0.12)$ \\
\hline \multicolumn{3}{|c|}{ Diffusion Markers (NODDI and DTI models) } \\
\hline Genu ISOVF & $0.11(0.03)$ & $0.13(0.03)$ \\
\hline Genu NDI & $0.54(0.04)$ & $0.52(0.04)$ \\
\hline ITWM NDI & $0.49(0.04)$ & $0.47(0.03)$ \\
\hline CGH NDI & $0.49(0.03)$ & $0.46(0.03)$ \\
\hline
\end{tabular}

CDR, clinical dementia rating scale; WMH, white matter hyperintensity; SUVR, standard uptake value ratio; TDP-43, trans-active response DNA-binding protein of 43; ISOVF, isotropic volume fraction; CGH, parahippocampal cingulum; ITWM, inferior temporal white matter, NDI, neurite density index

${ }^{a}$ Eighty-eight participants from MCSA and fourteen participants from ADRC were missing FDG PET scans

$P=0.02)$ in the MCSA, but none of the partial correlations were significant in the ADRC.

As stated in the introduction, both ITWM and CGH are likely damaged by tau and TDP- 43 such that loss of synapses and axons would be reflected in worsening neurite density on NODDI. Therefore, we considered these two tracts as our temporal WM component. In $\mathrm{ADRC}$, where there is a greater range of tau, we found evidence that neurite density (correlate of loss of synapses and neurons) in the ITWM was marginally associated with tau $(\mathrm{r}=-0.24, P=0.07$ for ITWM). This is illustrated in the middle panel of Fig. 1 . Using the FDG-PET TDP-43 signature, we found that reduced NDI in the CGH of MCSA $(n=259)$ was associated with increased FDG-PET TDP-43 signature $(r=-0.23$, $P<0.001$ for CGH NDI). This is shown in the third panel of Fig. 1. We also performed a sensitivity analysis 


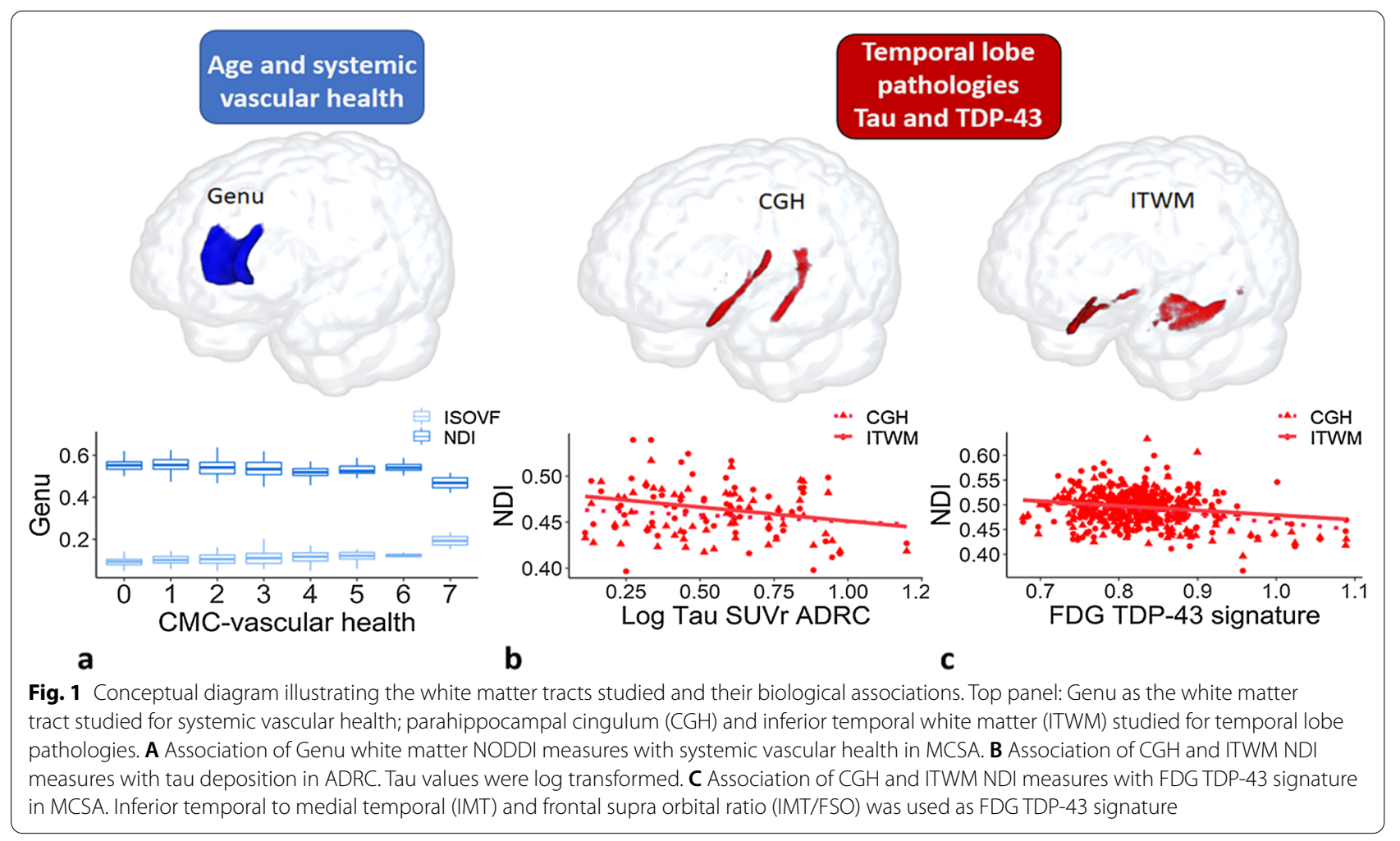

in a subset of amyloid negative $80+$ MCSA participants $(\mathrm{n}=22$ of which 17 were cognitively unimpaired and 5 were impaired), who are also likely to have low WM damage in association with tau and are at a high risk of TDP-43. We found a significant association of NDI in CGH with TDP-43 signature $(\mathrm{r}=-0.59, P=0.007)$. We did not find any associations of ISOVF in temporal tracts with disease biomarkers, so we did not consider ISOVF in the temporal WM component for further analyses.

\section{Relevance of NODDI in the context of AD biomarkers}

The models for both MCSA and ADRC samples showing an association between the individual neuroimaging measures and cognitive performance after adjusting for age, sex, education, and number of clinical visits (single biomarker models) are shown in Additional file 1: Table S1 and also in Fig. 2. To identify the contribution of WM NODDI measures to overall cognitive performance, we considered global cognition as a primary outcome in the MCSA. In MCSA, amyloid, tau, and all diffusion metrics showed a significant contribution to global cognition and MMSE when each factor was considered independently. Among the diffusion metrics, higher Genu ISOVF was most strongly associated with lower cognitive performance. We also observed a greater predictive power for Genu ISOVF (partial $R^{2}=6.9 \%$ for global cognition and 3.4\% for MMSE) than Genu NDI (partial $\mathrm{R}^{2}=3 \%$ for global cognition and 2.7\% for MMSE) in Fig. 2. Tau was a relatively lesser predictor of cognitive performance in this community dwelling cohort owing to its broad age ranges and relatively lower frequency of tau positivity.

In the ADRC cohort of amyloid positive individuals, higher ITWM NDI had a better prediction for lower cognitive performance than tau ( $14.8 \%$ and $11 \%$ respectively) univariately.

\section{NODDI and AD biomarkers for detection of possible etiology and prediction of cognitive performance}

The final models that included all imaging biomarkers (except FDG-PET TDP-43 signature), age, sex, education, and number of clinical visits for predicting cognitive performance are shown in Table 2. Older age and male sex were associated with worse global cognitive performance in the MCSA. In both MCSA the global cognition and MMSE models, higher education was associated with better cognition. There was contribution of amyloid, Genu ISOVF, and Genu NDI to worse global cognition and MMSE. However, amyloid contribution to global cognition (7.4\%) was comparable to MMSE (6.6\%). After accounting for amyloidosis, there was no significant contribution of the WM temporal component with both global cognition and MMSE. In the ADRC sample, only 


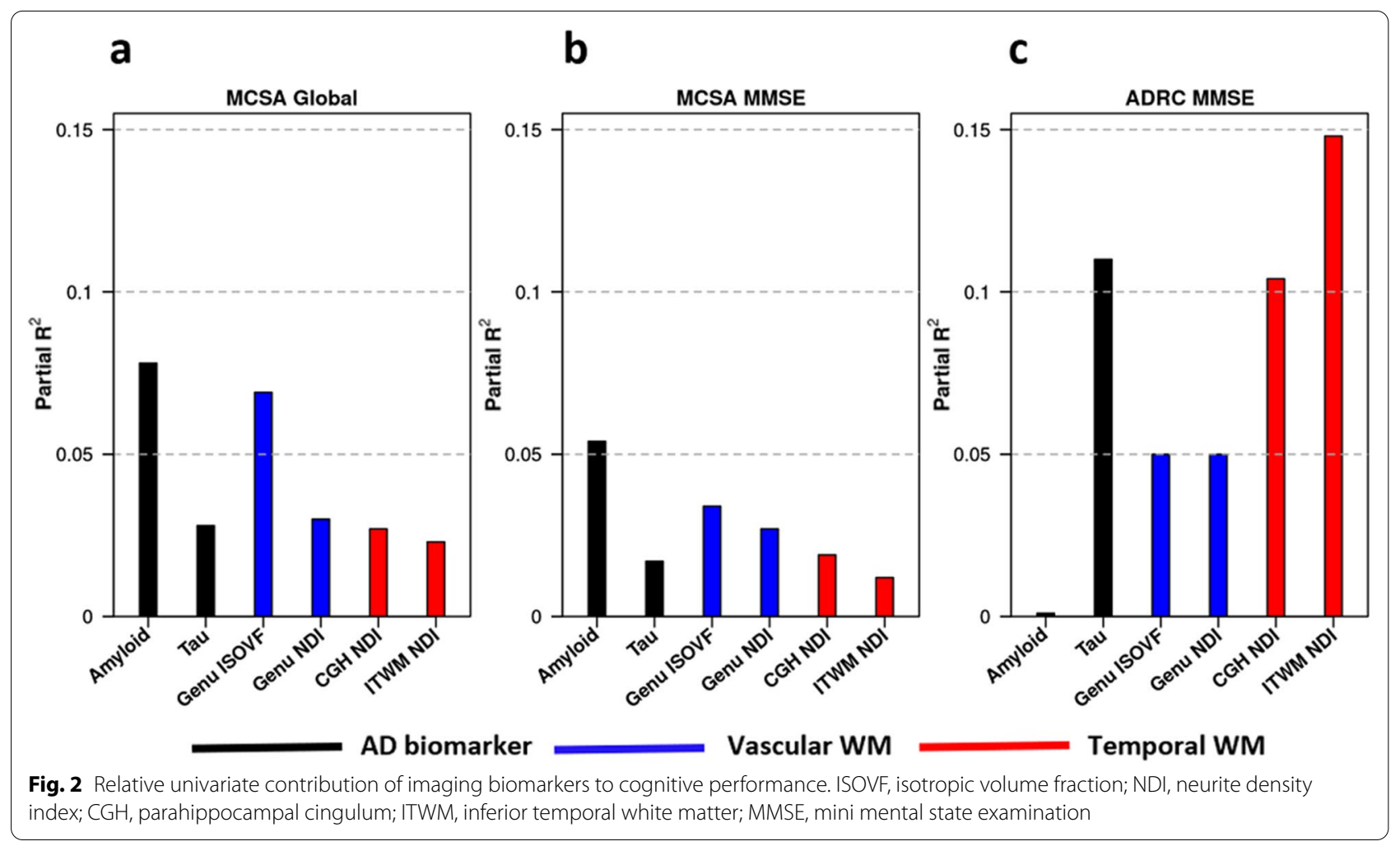

two predictors emerged as important. Lower tau (partial $\mathrm{R}^{2}=0.10$ ) and higher NDI in ITWM predicted better MMSE performance (partial $R^{2}=0.09$ ).

Figure 3 shows the predicted cognition by age group in MCSA and ADRC samples. Here, normal and abnormal were defined in quartiles with 25th and 75th percentiles of the measures within each age group respectively. For MCSA global cognition, the rapid lowering of predicted cognition as a function of age was expected given that age explains $12 \%$ of variance in cognition (Table 2) and the frequency of pathologies increases with age. For MCSA MMSE performance (middle panel), the average predicted MMSE was above 29 until age 70 after which there was a visible decline in the baseline MMSE which is typically observed in the population due to increasing burden of age-related pathologies. For the ADRC MMSE results in Fig. 3, there was worse MMSE in younger AD patients because more aggressive disease trajectories have been observed in younger AD patients [57].

\section{Neuropathology findings as indicators of TDP-43 status:}

Interestingly, a small subset $(\mathrm{n}=9)$ had autopsy findings. Though the samples are small, NODDI data compared against gold-standard pathology provides insights into variability of NODDI as a function of pathological burden specifically TDP-43. Three out of four MCSA cases $(75 \%)$ and two out of $5(40 \%)$ ADRC cases were
TDP-43(+). The imaging and neuropathological characteristics of the autopsy cohort are shown in Table 3 and discussed further below. The first TDP-43 positive case in MCSA (case 1) was hypertensive with a greater number of cardiovascular and metabolic conditions, higher Genu ISOVF, elevated IMT/FSO hypometabolism ratio, lower ITWM and CGH NDI reflecting the contribution of CVD and temporal WM contribution to impairment. Similarly, case 2 was tau-PET negative with hypertension, higher Genu ISOVF, high IMT/FSO hypometabolism ratio, very low ITWM and CGH NDI highlighting the usefulness of NODDI in helping assess vascular health. ADRC participants generally had low vascular disease burden but a greater extent of MTL damage (low Genu ISOVF, low ITWM, and low CGH NDI) which was reflected in the ADRC final model. Except one control, all other TDP43(+) cases had abnormal hippocampal volume.

\section{Discussion}

In this study, we assessed WM damage using NODDI measures in the frontal and temporal lobes and evaluated their contribution to cognitive decline in two independent data sets. The main conclusions were: (i) Both Genu ISOVF and Genu NDI were sensitive to capturing age and systemic vascular health related WM damage and contributed to the prediction of cognitive performance. 
Table 2 Final parsimonious models evaluating the utility of neuroimaging measures in predicting cognitive performance

\begin{tabular}{lccc}
\hline Variable & Estimate (s.e.) & $\boldsymbol{p}$ value & Partial $\mathbf{R}^{\mathbf{2}}$ \\
\hline MCSA Global Cognition & (Model $\left.R^{2}=0.546\right)$ & & \\
Intercept & $0.48(1.08)$ & 0.66 & \\
Age & $-0.06(0.009)$ & $<0.001$ & 0.121 \\
Male & $-0.23(0.10)$ & 0.031 & 0.014 \\
Education & $0.17(0.02)$ & $<0.001$ & 0.178 \\
Visit Number & $0.11(0.02)$ & $<0.001$ & 0.090 \\
Amyloid & $-1.23(0.24)$ & $<0.001$ & 0.074 \\
Genu ISOVF & $-9.16(2.20)$ & $<0.001$ & 0.049 \\
Genu NDI & $4.65(1.35)$ & $<0.001$ & 0.034 \\
MCSA MMSE (Model $\left.R^{2}=0.282\right)$ & & \\
Intercept & $23.26(1.35)$ & $<0.001$ & \\
Education & $0.19(0.03)$ & $<0.001$ & 0.092 \\
Amyloid & $-1.78(0.36)$ & $<0.001$ & 0.066 \\
Genu ISOVF & $-11.91(3.12)$ & $<0.001$ & 0.041 \\
Genu NDI & $8.01(2.09)$ & $<0.001$ & 0.041 \\
ADRCMMSE (Model $\left.R^{2}=0.209\right)$ & & \\
Intercept & $4.15(9.45)$ & 0.66 & \\
Tau & $-5.99(2.41)$ & 0.016 & 0.096 \\
ITWM NDI & $45.08(19.40)$ & 0.024 & 0.085 \\
\hline ISOVF isotropic & & & \\
\hline
\end{tabular}

ISOVF, isotropic volume fraction; CGH, parahippocampal cingulum; ITWM, inferior temporal white matter, NDI, neurite density index, MMSE, mini mental state examination. The initial had all NODDI measures but only these variables Significant predictors of cognition are shown in the parsimonious models. The models included all of these as potential predictors: age, male, education, visit number, amyloid, tau, genu ISOVF, genu NDI, ITWM NDI, and ITWM CGH

The utility of Genu ISOVF was greater in the populationbased cohort in comparison to the clinically diagnosed $\mathrm{AD}$ dementia cohort with elevated $\mathrm{A} \beta$ where there was lower vascular contribution to cognitive impairment. (ii) Neurite density measures in the temporal lobe tracts captured WM changes due to temporal lobe focused pathologies which was associated with tau PET and FDG-PET based TDP-43 signature. (iv) NODDI imaging acquired in the single setting of MRI would add value in assessing the possible etiology (CVD vs. tau related changes vs. possibly TDP-43 in the absence of amyloid and tau) and their corresponding contribution to cognitive performance.

\section{WM damage and CVD pathologies and its relevance for predicting cognition}

We and others have shown that vascular risk factors are associated with poor WM health [6, 21, 58], particularly reduced Genu FA with hypertension [58-60], diabetes [61, 62], and dyslipidemia [63]. However, the FA metric cannot accurately disentangle the contribution from tissue degeneration (axonal damage and myelin breakdown) and free water increase, and NODDI helped us separate these factors using NDI and ISOVF. Our recent study showed that white matter hyperintensities were the strongest predictors of diffusion changes (ISOVF) in addition to age [33]. In this study, we further report that Genu ISOVF and Genu NDI was associated with systemic vascular health in the population-based cohort. Additionally, previous studies observed increased free water changes in the normal-appearing WM using free water elimination method [64-66], suggesting that mechanisms of microvascular degeneration and neuroinflammation due to blood-brain barrier dysfunction contribute to mild vascular damage in small vessel disease and AD.

In the present study, we found a significant contribution of Genu ISOVF and Genu NDI on global cognitive performance and MMSE in the general population along with amyloid (Figs. 2, 3). Previous studies including ours $[6,10]$ have shown the association of reduced WM integrity in the Genu and worse cognitive performance [67-69]. Our previous study showed that NODDI derived free water or ISOVF in the corpus callosum was a significant predictor of cognitive performance highlighting its usefulness in capturing vascular contribution to cognitive impairment [33]. Notably, as shown in Figs. 2 and 3, Genu was not a significant predictor of MMSE in the AD cohort where the vascular pathology was comparatively less. Our findings support the utility of NODDI for capturing vascular contributions to cognition in populations where there is a greater prevalence of vascular disease contributing to cognitive impairment.

The relevance of NODDI measures for the diagnosis of vascular etiology can also be deduced from Table 3. Case 1 was an 85-year-old male with a clinical presentation of multi-domain MCI $(\mathrm{MMSE}=27)$ with amyloid and tau PET negative. Both the higher number of cardiovascular and metabolic conditions (4 out of 7 possible) and very high (or worse) Genu ISOVF of 0.22 (mean and standard deviation in MCSA were 0.11 and 0.03) and low Genu NDI of 0.49 (mean and standard deviation in MCSA were 0.54 and 0.04 ) indicates that the vascular contribution to impairment was significant and not sufficiently considered for diagnosis. Similarly, case 2, an 79-year-old male with amnestic MCI, was amyloid positive and tau PET negative. The high Genu ISOVF and low Genu NDI clearly supports the vascular contribution to cognitive impairment. Contrasting cases 5-8 from the ADRC, the lower values of Genu ISOVF and higher Genu NDI explain that the lower extent of vascular disease in the AD cases. While the selfreport of $\mathrm{CMC}$ was available, the continuous scale of Genu ISOVF reflects the true extent of biological damage.

\section{Temporal white matter damage and tau and its relevance for predicting cognition}

Tau pathology is the underlying cause of axonal and neuronal neurodegeneration in some cognitively normal and 


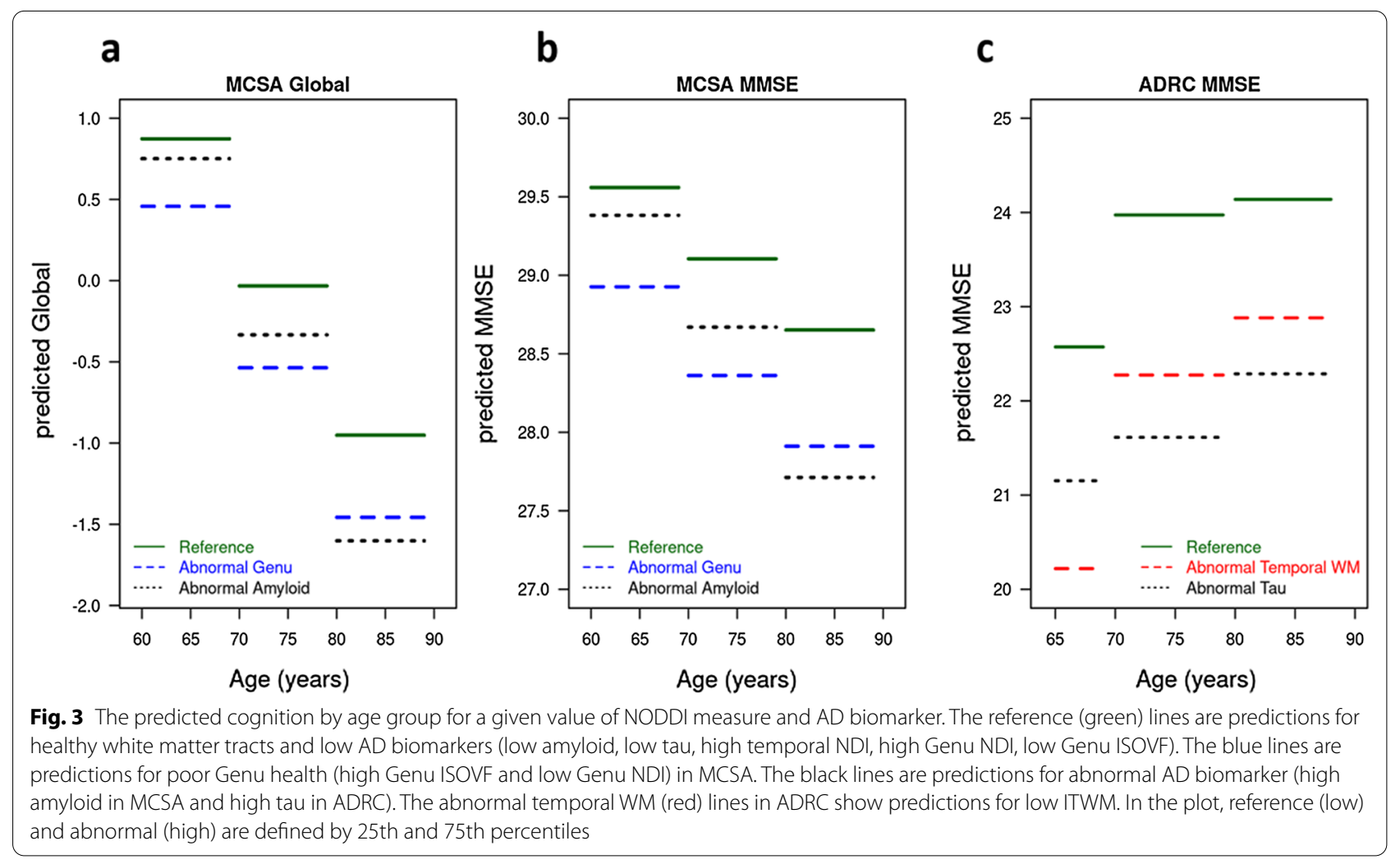

in $\mathrm{AD}$ dementia individuals $[12,14,70]$. Tau related WM changes often depicts distinct spatial profiles with initial accumulation in temporal lobe regions susceptible to $\mathrm{AD}$ pathology and then propagates through axonal projections. Evidence from tau-mouse models showed reduced NDI in WM and hippocampus [28]. In the present study, we showed that in a typical AD population with a greater extent of tau related changes, the temporal NDI (ITWM) measures were useful in detecting tau related diffusion changes. Here, we extend previous observations that reported an association between DTI and NFT pathology in the MTL limbic connections and medial parietal WM [12]. Evidence also suggests the association between tau PET and anterior temporal cortex and associated pathways [70], suggesting greater WM vulnerability in tracts associated with early tau accumulation $[29,71]$.

CGH is an important tract connecting the hippocampus to the posterior cingulate cortex, and it has been reported that $\mathrm{CGH}$ diffusivity significantly predicts downstream tau accumulation and associated memory decline in amyloid positive individuals [14]. More recently, Wen et al. [16] studied the spatial pattern of tau propagation using a DTI and NODDI derived datadriven method and suggested reduced axonal packing density and higher diffusion freedom in white matter in regions of tau deposition (which included inferior temporal to inferior parietal regions in amyloid positive participants). Additionally, studies have shown reduced cortical NDI in temporal and frontal lobes $[31,32]$ which is suggested to be associated with worse cognitive performance in $\mathrm{AD}[29,31,32]$. In our data, white matter temporal component NDI predicted MMSE and global cognitive performance, suggesting that these regional metrics could be sensitive to tau mediated WM damage and hence would be a relevant biomarker for studying disease progression. When we focused on multivariable analyses using a combination of Lasso and stepwise regression, tau and ITWM NDI emerged as the significant predictors of cognitive performance for typical AD. This was supported by recent in vivo PET studies that demonstrated a more extensive reduction in synaptic density in the medial temporal and neocortical early AD brains [72, 73]. Furthermore, reduced cortical NDI and ODI in the mesial and lateral temporal lobes of AD brains were associated with increased ${ }^{18} \mathrm{~F}$-THK5351 signal and worse cognitive performance, implicating the role of tau and neuroinflammatory pathology in the formation of abnormal neuritis [74]. Notably, our observed non-trivial contribution of the NDI in ITWM (early neocortical pathway associated to tau deposition in $\mathrm{AD}$ ) to cognitive performance along with tau suggests the integral role of NODDI as 


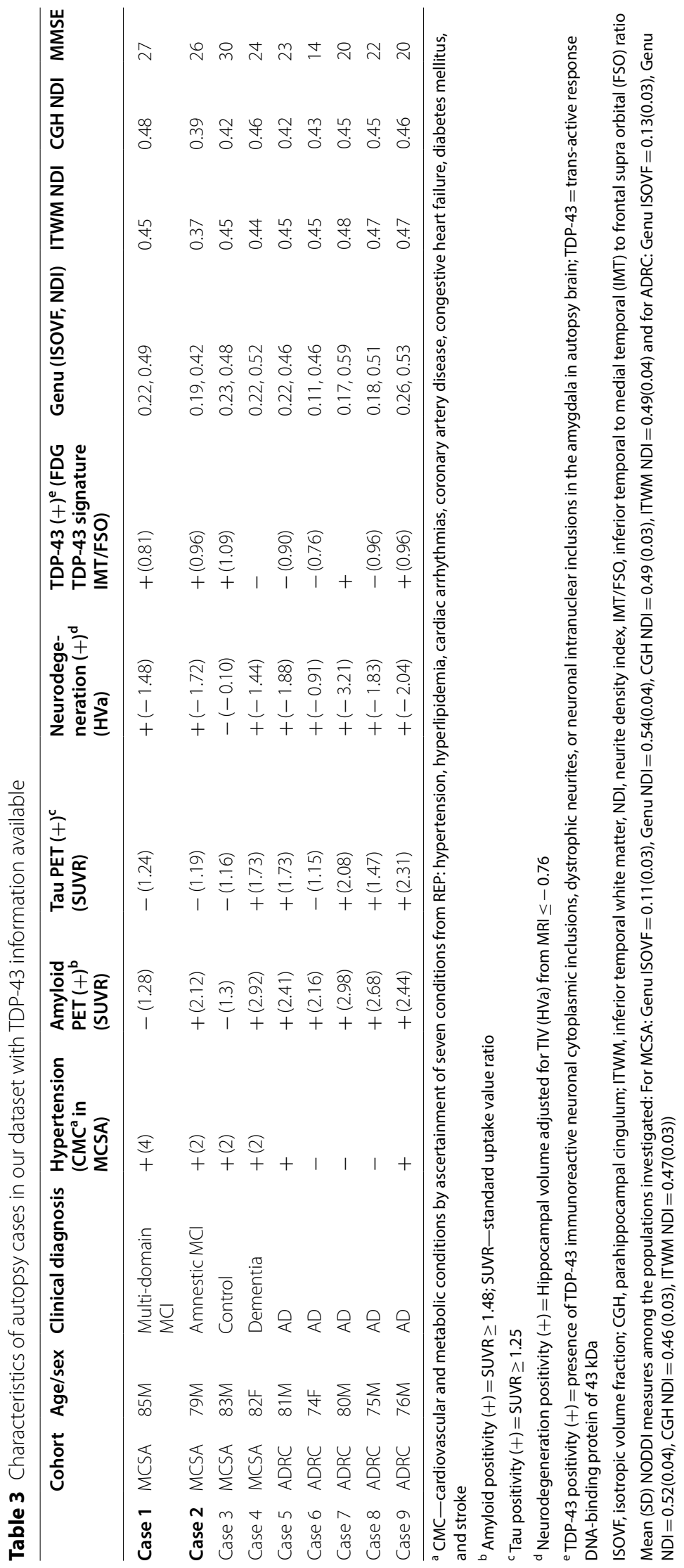


an early marker of cognitive changes in the preclinical stages (clearly shown in the third panel of Fig. 3)

Amyloid was useful in predicting cognition in the community cohort because there is a greater range of amyloidosis across the population and lower frequency of high levels of tau burden. However, the multivariable analysis in the MCSA not retained the effect of NDI in temporal tracts suggesting that temporal WM health is more strongly associated with tau than vascular etiology (Fig. 2). On the other hand, tau PET information and tau-mediated white matter injury (reflected with worsening ITWM NDI) predicted memory performance in the ADRC. Figure 3 illustrates how specific NODDI measures can be used to predict cognition along with $\mathrm{AD}$ biomarkers in two independent populations and the extent of damage on NODDI can also reflect the extent of damage attributed to CVD vs. tau-specific damage.

\section{Temporal white matter damage and TDP-43 and its relevance for predicting cognition}

The co-existence of multiple pathologies along with $\mathrm{AD}$ neuropathological changes especially in individuals after 80 years of age adds to the prediction of worsening cognitive performance. Clinical and neuropathological evidence identified accumulation of TDP-43 inclusions in association with $\mathrm{AD}[2,3]$ and hippocampal sclerosis [75], suggesting it is a major contributor to cognitive dysfunction. Also, TDP-43 exacerbates the memory impairment and cognitive decline in individuals with $\mathrm{AD}$ pathologies $[3,76]$. While there is no definite biomarker for TDP-43 other than autopsy, predominant involvement of MTL structures $[4,5]$ and recent evidence supports the usefulness of a FDG-PET hypometabolic signature as being correlated with the presence of TDP-43 $[18,19]$. This proposed FDG TDP-43 marker, the derived IMT or IMT/ FSO ratio, reliably identified the tau-negative amnestic status in older individuals $[18,19]$. The association we observed between CGH NDI and FDG IMT/FSO ratio in all MCSA and amyloid negative 80 plus individuals suggests that neurite density also changes with other prominent non-AD temporal lobe pathology such as TDP-43.

Pathology information sheds additional light on the relevance of NODDI temporal WM values for identifying the likely etiology in Table 3 . Cases $1-3$ were classified as TDP-43(+) at autopsy. In all three cases, the tau PET scans were also negative, but the temporal WM measure was abnormal because the values were lower than 0.49 (average for CGH and ITWM in MCSA). The worse NDI in these tracts that are spread across the temporal lobes illustrates the likelihood that a different etiology such as TDP-43 or PART [77] (primary age-related tauopathy refers tau in the absence of amyloidosis) may have contributed to the cognitive impairment. As reported previously $[18,78]$, the current TDP-43(+) participants had abnormal hippocampal volumes, suggesting that both TDP-43 and hippocampal sclerosis accounted the tau-negative amnestic syndrome. While the NODDI measures also have the same non-specificity of a medial temporal atrophy [79] measure, future studies in larger datasets with autopsy confirmed data will be able to shed light on the usefulness of NODDI for identifying the underlying etiology of cognitive impairment. It cannot be ruled out that the WM changes observed may be due to PART, which will need confirmation in a larger autopsy cohort.

\section{Usefulness of NODDI in Aging and Dementia Studies}

Overall, our findings support the utility of non-invasive, increasingly available, and affordable biophysical diffusion models as proxy measures for WM damage due to vascular, $\mathrm{AD}$, and TDP-43. As multi-band acceleration has become commonplace, these measures can inform the likely substrate of cognitive impairment and can facilitate early detection and tracking of disease progression in aging and dementia studies. Future studies will build on this work to optimize the usage of NODDI for differential diagnosis and expand on the idea of tracking longitudinal WM damage with disease progression.

An important finding from this study is that the usefulness of regional NODDI measures will vary based on the cohort under investigation. For example, in the community-dwelling cohort where multiple pathologies increase with age and contribute to cognitive decline, frontal WM would contribute to the prediction of cognitive performance. On the other hand, in clinically diagnosed AD dementia patients recruited from a dementia clinic where the primary substrate of dementia is likely tau deposition, temporal WM damage related to tau would be a significant predictor of cognitive performance.

\section{Strength and limitations}

A key strength of this study was the rich multimodal imaging information from two independent samples with varying degrees of CVD and $A D$ pathologies along with pathology information in a subset. This allowed us to assess the vascular and $\mathrm{AD}$ contributions to diffusion alterations and associated cognitive performance. The use of an advanced diffusion model with conventional DTI enabled us to disentangle the source of WM alterations more accurately with precise control of CSF partial volume effects. Another strength is the focus on multiple cognitive measures in the MCSA sample to evaluate CVD-related cognitive impairment. A major limitation is the relatively modest sample size of typical AD participants in ADRC with comparatively less vascular disease that may limit the tract specific association findings. 
Another limitation is that we utilized FDG-PET TDP-43 signature as a surrogate for TDP-43 due to the lack of sufficient pathology data and TDP-43 also may likely be present in AD patients. However the association between NODDI and the FDG-PET TDP-43 signature in amyloid negative $80+$ participants lends support for our hypothesis that NDI in temporal WM tracts are impacted by TDP-43. Future work based on a larger cohort with multiple biophysical diffusion models, pathology information, and longitudinal imaging measurements will further allow us to expand on these important findings.

\section{Conclusions}

In the present study, we found that cerebrovascular disease, tau, and TDP-43 proteinopathy cause white matter microstructural damage measurable using advanced diffusion NODDI models. In two independent cohorts with different clinical composition, we showed the clinical utility of NODDI models in predicting cognitive performance and its usefulness in identifying the substrate of cognitive impairment. Therefore, NODDI, acquired in a single setting of MRI, can add significant clinical value for differential diagnosis in cognitive impairment and dementia.

\begin{abstract}
Abbreviations
AD: Alzheimer's disease; ADRC: Alzheimer's Disease Research Center; CGH: Parahippocampal cingulum; CSF: Cerebrospinal fluid; CU: Cognitively unimpaired; CVD: Cerebrovascular disease; DTI: Diffusion tensor imaging; FA: Fractional anisotropy; FDG-PET: Fluorodeoxyglucose PET (FDG-PET); FLAIR: Fluid attenuated inversion recovery; GCC: Genu of corpus callosum; GM: Gray matter; ISOVF: Isotropic volume fraction; ITWM: Inferior temporal white matter; MCl: Mild cognitive impairment; MCSA: Mayo clinic study of aging; MD: Mean diffusivity; MMSE: Mini mental state examination; NDI: Neurite density index; NODDI: Neurite orientation dispersion and density Imaging; ODI: Orientation dispersion index; PET: Positron emission tomography; TDP-43: TAR DNA binding protein of $43 \mathrm{kDa}$; WM: White matter.
\end{abstract}

\section{Supplementary Information}

The online version contains supplementary material available at https://doi. org/10.1186/s40478-022-01319-6.

Additional file 1: Table S1. Single biomarker models evaluating the utility of neuroimaging measures for predicting cognitive performance after accounting for age, sex, education, and number of clinical visits.

\section{Acknowledgements}

We thank all the study participants and staff in the Mayo Clinic Study of Aging, Mayo Alzheimer's Disease Research Center, and Aging Dementia Imaging Research laboratory at the Mayo Clinic for making this study possible. We gratefully acknowledge the support of NVIDIA Corporation for the donation of the Quadro P5000 GPU used in this research.

\section{Authors' contributions}

SKR and PV conceived and designed the study. All authors participated in data collection and analysis. SKR, PV, SAP, TGL and RIR drafted the manuscript and figures. All authors read and approved the final manuscript.

\section{Funding}

This work was supported by NIH grants R01 NS097495 (PI:Vemuri), U01 AG06786 (PI: Petersen/Mielke/Jack), R01 AG56366 (PI: Vemuri), P50 AG16574 (PI: Petersen), R37 AG11378 (PI: Jack), R01 AG41851 (PIs: Jack and Knopman), R01 AG054449 (PI: Murray); R01 AG37491 (PI Josephs); the Gerald and Henrietta Rauenhorst Foundation grant, Alzheimer's Drug Discovery Foundation (ADDF), the Alexander Family Alzheimer's Disease Research Professorship of the Mayo Foundation, Liston Award, Elsie and Marvin Dekelboum Family Foundation, Schuler Foundation, Opus building NIH grant C06 RR018898, and was made possible by Rochester Epidemiology Project (R01 AG34676).

\section{Availability of data and materials}

The data used in this study will be made available upon reasonable request following MCSA and ADRC study procedures.

\section{Declarations}

Ethics approval and consent to participate

The study was approved by the Mayo Clinic and Olmsted Medical Center institutional review boards and written informed consent was obtained from all participants or their qualified caregivers.

\section{Consent for publication}

Not applicable.

\section{Competing interests}

The authors report no competing interests related to the submitted manuscript. Dr. Raghavan reports no relevant financial disclosures. Mr. Przybelski reports no relevant financial disclosures. Dr. Reid reports no relevant financial disclosures. Mr. Lesnick reports no relevant financial disclosures. Dr. Ramanan reports no relevant financial disclosures. Dr. Hugo reports no relevant financial disclosures. Dr. Melissa reports no relevant financial disclosures. Dr. Knopman serves on a Data Safety Monitoring Board for the DIAN study. He serves on a Data Safety monitoring Board for Biogen, but receives no personal compensation. He is an investigator in clinical trials sponsored by Biogen, Lilly Pharmaceuticals, and the University of Southern California. He serves as a consultant for Roche, Samus Therapeutics, Third Rock and Alzeca Biosciences but receives no personal compensation. Dr. Graff-Radford serves as an assistant editor for Neurology and receives research support from the NIH. Dr. Jones receives research support from NIH. Mrs. Matchett reports no relevant financial disclosures. Dr. Lowe consults for Bayer Schering Pharma, Piramal Life Sciences, Life Molecular Imaging, Eisai Inc., AVID Radiopharmaceuticals, and Merck Research and receives research support from GE Healthcare, Siemens Molecular Imaging, AVID Radiopharmaceuticals, and the NIH (NIA, NCI). Dr. Mielke serves as a consultant for Biogen and Brain Protection Company and receives research funds from NIH and DOD. Dr. Machulda receives research support from NIH. Dr. Petersen serves as a consultant for Roche Inc., Merck Inc., and Biogen, Inc. He serves on the Data Safety monitoring Board for Genentech, Inc and receives royalty from Oxford University Press and UpToDate. Dr. Kantarci served on the data safety monitoring board for Takeda Global Research and Development Center, Inc;; receives research support from Avid Radioparmaceuticals and Eli Lilly, and receives funding from $\mathrm{NIH}$ and Alzheimer's Drug Discovery Foundation. Dr. Whitwell receives research support from NIH. Dr. Josephs receives research support from NINDS, NIA and NIDCD. Dr. Jack serves on an independent data monitoring board for Roche, has served as a speaker for Eisai, and consulted for Biogen, but he receives no personal compensation from any commercial entity. He receives research support from $\mathrm{NIH}$ and the Alexander Family Alzheimer's Disease Research Professorship of the Mayo Clinic. Dr. Vemuri received speaker fees from Miller Medical Communications, Inc. and receives research support from the $\mathrm{NIH}$.

\section{Author details}

'Department of Radiology, Mayo Clinic, 200 First Street SW, Rochester, MN 55905, USA. ${ }^{2}$ Department of Quantitative Health Sciences, Mayo Clinic, Rochester, MN 55905, USA. ${ }^{3}$ Department of Information Technology, Mayo Clinic, Rochester, MN 55905, USA. ${ }^{4}$ Department of Neurology, Mayo Clinic, Rochester, MN 55905, USA. ${ }^{5}$ Department of Neuroscience, Mayo Clinic, Jacksonville, FL 32224, USA. ${ }^{6}$ Department of Laboratory Medicine and Pathology, Mayo Clinic, Rochester, MN 55905, USA. ${ }^{7}$ Department of Psychiatry and Psychology, Mayo Clinic, Rochester, MN 55905, USA. 
Received: 14 January 2022 Accepted: 19 January 2022

Published online: 05 February 2022

\section{References}

1. Schneider JA, Arvanitakis Z, Leurgans SE, Bennett DA (2009) The neuropathology of probable Alzheimer disease and mild cognitive impairment. Ann Neurol 66(2):200-208

2. Josephs KA, Whitwell JL, Knopman DS, Hu WT, Stroh DA, Baker M et al (2008) Abnormal TDP-43 immunoreactivity in AD modifies clinicopathologic and radiologic phenotype. Neurology 70(19 Pt 2):1850-1857

3. Josephs KA, Whitwell JL, Weigand SD, Murray ME, Tosakulwong N, Liesinger AM et al (2014) TDP-43 is a key player in the clinical features associated with Alzheimer's disease. Acta Neuropathol 127(6):811-824

4. Josephs KA, Martin PR, Weigand SD, Tosakulwong N, Buciuc M, Murray ME et al (2020) Protein contributions to brain atrophy acceleration in Alzheimer's disease and primary age-related tauopathy. Brain 143(11):3463-3476

5. Nelson PT, Dickson DW, Trojanowski JQ, Jack CR, Boyle PA, Arfanakis K et a (2019) Limbic-predominant age-related TDP-43 encephalopathy (LATE): consensus working group report. Brain 142(6):1503-1527

6. Vemuri P, Lesnick TG, Przybelski SA, Graff-Radford J, Reid RI, Lowe VJ et al (2018) Development of a cerebrovascular magnetic resonance imaging biomarker for cognitive aging. Ann Neurol 84(5):705-716

7. Wassenaar TM, Yaffe K, van der Werf YD, Sexton CE (2019) Associations between modifiable risk factors and white matter of the aging brain: insights from diffusion tensor imaging studies. Neurobiol Aging 80:56-70

8. Bennett IJ, Madden DJ (2014) Disconnected aging: cerebral white matter integrity and age-related differences in cognition. Neuroscience 276:187-205

9. Tullberg M, Fletcher E, DeCarli C, Mungas D, Reed BR, Harvey DJ et al (2004) White matter lesions impair frontal lobe function regardless of their location. Neurology 63(2):246-253

10. Raghavan S, Przybelski SA, Reid RI, Graff-Radford J, Lesnick TG, Zuk SM et al (2020) Reduced fractional anisotropy of the genu of the corpus callosum as a cerebrovascular disease marker and predictor of longitudinal cognition in MCl. Neurobiol Aging 96:176-183

11. Alzheimer A, Förstl H, Levy R (1991) On certain peculiar diseases of old age. Hist Psychiatry 2(5 Pt 1):71-101

12. Kantarci K, Murray ME, Schwarz CG, Reid RI, Przybelski SA, LesnickT et al (2017) White-matter integrity on DTI and the pathologic staging of Alzheimer's disease. Neurobiol Aging 56:172-179

13. Rabin JS, Yang HS, Schultz AP, Hanseeuw BJ, Hedden T, Viswanathan A et al (2019) Vascular risk and $\beta$-amyloid are synergistically associated with cortical tau. Ann Neurol 85(2):272-279

14. Jacobs HIL, Hedden T, Schultz AP, Sepulcre J, Perea RD, Amariglio RE et al (2018) Structural tract alterations predict downstream tau accumulation in amyloid-positive older individuals. Nat Neurosci 21(3):424-431

15. Tracy TE, Gan L (2018) Tau-mediated synaptic and neuronal dysfunction in neurodegenerative disease. Curr Opin Neurobiol 51:134-138

16. Wen Q, Risacher SL, Xie L, Li J, Harezlak J, Farlow MR et al (2021) Taurelated white-matter alterations along spatially selective pathways. Neuroimage 226:117560

17. Johnson KA, Schultz A, Betensky RA, Becker JA, Sepulcre J, Rentz D et al (2016) Tau positron emission tomographic imaging in aging and early Alzheimer disease. Ann Neurol 79(1):110-119

18. Botha H, Mantyh WG, Murray ME, Knopman DS, Przybelski SA, Wiste HJ et al (2018) FDG-PET in tau-negative amnestic dementia resembles that of autopsy-proven hippocampal sclerosis. Brain 141(4):1201-1217

19. Buciuc M, Botha H, Murray ME, Schwarz CG, Senjem ML, Jones DT et al (2020) Utility of FDG-PET in diagnosis of Alzheimer-related TDP-43 proteinopathy. Neurology 95(1):e23-e34

20. Caballero MÁA, Song Z, Rubinski A, Duering M, Dichgans M, Park DC et al (2020) Age-dependent amyloid deposition is associated with white matter alterations in cognitively normal adults during the adult life span. Alzheimer's Dementia 16(4):651-661

21. Cox SR, Lyall DM, Ritchie SJ, Bastin ME, Harris MA, Buchanan CR et al (2019) Associations between vascular risk factors and brain MRI indices in UK Biobank. Eur Heart J 40(28):2290-2300
22. Pines AR, Cieslak M, Larsen B, Baum GL, Cook PA, Adebimpe A et al (2020) Leveraging multi-shell diffusion for studies of brain development in youth and young adulthood. Dev Cognit Neurosci 43:100788

23. Schilling KG, Janve V, Gao Y, Stepniewska I, Landman BA, Anderson AW (2018) Histological validation of diffusion MRI fiber orientation distributions and dispersion. Neuroimage 165:200-221

24. Zhang H, Schneider T, Wheeler-Kingshott CA, Alexander DC (2012) NODDI: practical in vivo neurite orientation dispersion and density imaging of the human brain. Neuroimage 61(4):1000-1016

25. Kodiweera C, Alexander AL, Harezlak J, McAllister TW, Wu YC (2016) Age effects and sex differences in human brain white matter of young to middle-aged adults: A DTI, NODDI, and q-space study. Neuroimage 128:180-192

26. Cox SR, Ritchie SJ, Tucker-Drob EM, Liewald DC, Hagenaars SP, Davies G et al (2016) Ageing and brain white matter structure in 3,513 UK Biobank participants. Nat Commun 7:13629

27. Kamagata K, Zalesky A, Hatano T, Ueda R, Di Biase MA, Okuzumi A et al (2017) Gray matter abnormalities in idiopathic Parkinson's disease: evaluation by diffusional kurtosis imaging and neurite orientation dispersion and density imaging. Hum Brain Mapp 38(7):3704-3722

28. Colgan N, Siow B, O'Callaghan JM, Harrison IF, Wells JA, Holmes HE et al (2016) Application of neurite orientation dispersion and density imaging (NODDI) to a tau pathology model of Alzheimer's disease. Neuroimage 125:739-744

29. Fu X, Shrestha S, Sun M, Wu Q, Luo Y, Zhang X et al (2019) Microstructural white matter alterations in mild cognitive impairment and Alzheimer's disease: study based on neurite orientation dispersion and density imaging (NODDI). Clin Neuroradiol 30(3):569-579

30. Wen Q, Mustafi SM, Li J, Risacher SL, Tallman E, Brown SA et al (2019) White matter alterations in early-stage Alzheimer's disease: a tract-specific study. Alzheimers Dement (Amst) 11:576-587

31. Parker TD, Slattery CF, Zhang J, Nicholas JM, Paterson RW, Foulkes AJM et al (2018) Cortical microstructure in young onset Alzheimer's disease using neurite orientation dispersion and density imaging. Hum Brain Mapp 39(7):3005-3017

32. Vogt NM, Hunt JF, Adluru N, Dean DC, Johnson SC, Asthana S et al (2020) Cortical microstructural alterations in mild cognitive impairment and Alzheimer's disease dementia. Cereb Cortex 30(5):2948-2960

33. Raghavan S, Reid RI, Przybelski SA, Lesnick TG, Graff-Radford J, Schwarz CG et al (2021) Diffusion models reveal white matter microstructural changes with ageing, pathology and cognition. Brain Commun 3(2):fcab106

34. Roberts RO, Geda YE, Knopman DS, Cha RH, Pankratz VS, Boeve BF et al (2008) The Mayo Clinic Study of Aging: design and sampling, participation, baseline measures and sample characteristics. Neuroepidemiology 30(1):58-69

35. Rocca WA, Yawn BP, St Sauver JL, Grossardt BR, Melton LJ 3rd (2012) History of the Rochester Epidemiology Project: half a century of medical records linkage in a US population. Mayo Clin Proc 87(12):1202-1213

36. St Sauver JL, Grossardt BR, Yawn BP, Melton LJ 3rd, Pankratz JJ, Brue SM et al (2012) Data resource profile: the Rochester Epidemiology Project (REP) medical records-linkage system. Int J Epidemiol 41(6):1614-1624

37. Petersen RC, Roberts RO, Knopman DS, Geda YE, Cha RH, Pankratz VS et al (2010) Prevalence of mild cognitive impairment is higher in men. The Mayo Clinic Study of Aging. Neurology 75(10):889-897

38. Vemuri P, Lesnick TG, Przybelski SA, Knopman DS, Lowe VJ, Graff-Radford J et al (2017) Age, vascular health, and Alzheimer disease biomarkers in an elderly sample. Ann Neurol 82(5):706-718

39. Caruyer E, Lenglet C, Sapiro G, Deriche R (2013) Design of multishell sampling schemes with uniform coverage in diffusion MRI. Magn Reson Med 69(6):1534-1540

40. Veraart J, Novikov DS, Christiaens D, Ades-Aron B, Sijbers J, Fieremans E (2016) Denoising of diffusion MRI using random matrix theory. Neuroimage 142:394-406

41. Andersson JLR, Sotiropoulos SN (2016) An integrated approach to correction for off-resonance effects and subject movement in diffusion MR imaging. Neuroimage 125:1063-1078

42. Kellner E, Dhital B, Kiselev VG, Reisert M (2016) Gibbs-ringing artifact removal based on local subvoxel-shifts. Magn Reson Med 76(5):1574-1581 
43. Koay CG, Ozarslan E, Basser PJ (2009) A signal transformational framework for breaking the noise floor and its applications in MRI. J Magn Resonance (San Diego, Calif: 1997) 197(2):108-119

44. Garyfallidis E, Brett M, Amirbekian B, Rokem A, van der Walt S, Descoteaux M et al (2014) Dipy, a library for the analysis of diffusion MRI data. Front Neuroinform 8:8

45. Daducci A, Canales-Rodríguez EJ, Zhang H, Dyrby TB, Alexander DC, Thiran JP (2015) Accelerated microstructure imaging via convex optimization (AMICO) from diffusion MRI data. Neuroimage 105:32-44

46. Oishi K, Faria A, Jiang H, Li X, Akhter K, Zhang J et al (2009) Atlas-based whole brain white matter analysis using large deformation diffeomorphic metric mapping: application to normal elderly and Alzheimer's disease participants. Neuroimage 46(2):486-499

47. Avants BB, Tustison NJ, Song G, Cook PA, Klein A, Gee JC (2011) A reproducible evaluation of ANTs similarity metric performance in brain image registration. Neuroimage 54(3):2033-2044

48. Jack CR Jr, Wiste HJ, Weigand SD, Therneau TM, Lowe VJ, Knopman DS et al (2017) Defining imaging biomarker cut points for brain aging and Alzheimer's disease. Alzheimer's Dementia 13(3):205-216

49. Mirra SS, Heyman A, McKeel D, Sumi SM, Crain BJ, Brownlee LM et al (1991) The Consortium to Establish a Registry for Alzheimer's Disease (CERAD). Part II. Standardization of the neuropathologic assessment of Alzheimer's disease. Neurology 41(4):479-486

50. Lin WL, Castanedes-Casey M, Dickson DW (2009) Transactivation response DNA-binding protein 43 microvasculopathy in frontotemporal degeneration and familial Lewy body disease. J Neuropathol Exp Neurol 68(11):1167-1176

51. Josephs KA, Murray ME, Whitwell JL, Tosakulwong N, Weigand SD, Petrucelli L et al (2016) Updated TDP-43 in Alzheimer's disease staging scheme. Acta Neuropathol 131(4):571-585

52. Vemuri P, LesnickTG, Przybelski SA, Machulda M, Knopman DS, Mielke MM et al (2014) Association of lifetime intellectual enrichment with cognitive decline in the older population. JAMA Neurol 71(8):1017-1024

53. Folstein MF, Folstein SE, McHugh PR (1975) "Mini-mental state". A practical method for grading the cognitive state of patients for the clinician. J Psychiatr Res 12(3):189-198

54. Kokmen E, Smith GE, Petersen RC, Tangalos E, Ivnik RC (1991) The short test of mental status. Correlations with standardized psychometric testing. Arch Neurol 48(7):725-728

55. Rothman KJ (1990) No adjustments are needed for multiple comparisons. Epidemiology 1(1):43-46

56. Perneger TV (1998) What's wrong with Bonferroni adjustments. BMJ 316(7139):1236-1238

57. La Joie R, Visani AV, Baker SL, Brown JA, Bourakova V, Cha J et al (2020) Prospective longitudinal atrophy in Alzheimer's disease correlates with the intensity and topography of baseline tau-PET. Sci Transl Med 12:524

58. Duering M, Gesierich B, Seiler S, Pirpamer L, Gonik M, Hofer E et al (2014) Strategic white matter tracts for processing speed deficits in age-related small vessel disease. Neurology 82(22):1946-1950

59. Maillard P, Seshadri S, Beiser A, Himali JJ, Au R, Fletcher E et al (2012) Effects of systolic blood pressure on white-matter integrity in young adults in the Framingham Heart Study: a cross-sectional study. Lancet Neurol 11(12):1039-1047

60. Salat DH, Williams VJ, Leritz EC, Schnyer DM, Rudolph JL, Lipsitz LA et al (2012) Inter-individual variation in blood pressure is associated with regional white matter integrity in generally healthy older adults. Neuroimage 59(1):181-192

61. Falvey CM, Rosano C, Simonsick EM, Harris T, Strotmeyer ES, Satterfield S et al (2013) Macro- and microstructural magnetic resonance imaging indices associated with diabetes among community-dwelling older adults. Diabetes Care 36(3):677-682

62. Hsu JL, Chen YL, Leu JG, Jaw FS, Lee CH, Tsai YF et al (2012) Microstructural white matter abnormalities in type 2 diabetes mellitus: a diffusion tensor imaging study. Neuroimage 59(2):1098-1105

63. Cohen Jl, Cazettes F, Convit A (2011) Abnormal cholesterol is associated with prefrontal white matter abnormalities among obese adults, a diffusion tensor imaging study. Neuroradiol J 1(21):989-997

64. Maillard P, Mitchell GF, Himali JJ, Beiser A, Fletcher E, Tsao CW et al (2017) Aortic stiffness, increased white matter free water, and altered microstructural integrity: a continuum of injury. Stroke 48(6):1567-1573
65. Duering M, Finsterwalder S, Baykara E, Tuladhar AM, Gesierich B, Konieczny MJ et al (2018) Free water determines diffusion alterations and clinical status in cerebral small vessel disease. Alzheimers Dement 14(6):764-774

66. Ji F, Pasternak O, Liu S, Loke YM, Choo BL, Hilal S et al (2017) Distinct white matter microstructural abnormalities and extracellular water increases relate to cognitive impairment in Alzheimer's disease with and without cerebrovascular disease. Alzheimers Res Ther 9(1):63

67. Tuladhar AM, van Norden AG, de Laat KF, Zwiers MP, van Dijk EJ, Norris DG et al (2015) White matter integrity in small vessel disease is related to cognition. Neurolmage Clinical 7:518-524

68. Jokinen H, Ryberg C, Kalska H, Ylikoski R, Rostrup E, Stegmann MB et al (2007) Corpus callosum atrophy is associated with mental slowing and executive deficits in subjects with age-related white matter hyperintensities: the LADIS Study. J Neurol Neurosurg Psychiatry 78(5):491-496

69. Román GC, Erkinjuntti T, Wallin A, Pantoni L, Chui HC (2002) Subcortical ischaemic vascular dementia. Lancet Neurol 1(7):426-436

70. Strain JF, Smith RX, Beaumont H, Roe CM, Gordon BA, Mishra S et al (2018) Loss of white matter integrity reflects tau accumulation in Alzheimer disease defined regions. Neurology 91(4):e313-e318

71. Mito R, Raffelt D, Dhollander T, Vaughan DN, Tournier JD, Salvado O et al (2018) Fibre-specific white matter reductions in Alzheimer's disease and mild cognitive impairment. Brain 141(3):888-902

72. Mecca AP, Chen MK, O'Dell RS, Naganawa M, Toyonaga T, Godek TA et al (2020) In vivo measurement of widespread synaptic loss in Alzheimer's disease with SV2A PET. Alzheimers Dement 16(7):974-982

73. Bastin C, Bahri MA, Meyer F, Manard M, Delhaye E, Plenevaux A et al (2020) In vivo imaging of synaptic loss in Alzheimer's disease with [18F] UCB-H positron emission tomography. Eur J Nucl Med Mol Imaging 47(2):390-402

74. Sone D, Shigemoto Y, Ogawa M, Maikusa N, Okita K, Takano H et al (2020) Association between neurite metrics and tau/inflammatory pathology in Alzheimer's disease. Alzheimers Dement (Amst) 12(1):e12125

75. Nelson PT, Head E, Schmitt FA, Davis PR, Neltner JH, Jicha GA et al (2011) Alzheimer's disease is not "brain aging": neuropathological, genetic, and epidemiological human studies. Acta Neuropathol 121(5):571-587

76. Josephs KA, Whitwell JL, Tosakulwong N, Weigand SD, Murray ME, Liesinger AM et al (2015) TAR DNA-binding protein 43 and pathological subtype of Alzheimer's disease impact clinical features. Ann Neurol 78(5):697-709

77. Crary JF, Trojanowski JQ, Schneider JA, Abisambra JF, Abner EL, Alafuzoff I et al (2014) Primary age-related tauopathy (PART): a common pathology associated with human aging. Acta Neuropathol 128(6):755-766

78. Botha H, Mantyh WG, Graff-Radford J, Machulda MM, Przybelski SA, Wiste $\mathrm{HJ}$ et al (2018) Tau-negative amnestic dementia masquerading as Alzheimer disease dementia. Neurology 90(11):e940-e946

79. de Flores R, Wisse LEM, Das SR, Xie L, McMillan CT, Trojanowski JQ et al (2020) Contribution of mixed pathology to medial temporal lobe atrophy in Alzheimer's disease. Alzheimers Dement 16(6):843-852

\section{Publisher's Note}

Springer Nature remains neutral with regard to jurisdictional claims in published maps and institutional affiliations.

$$
\begin{aligned}
& \text { Ready to submit your research? Choose BMC and benefit from: } \\
& \text { - fast, convenient online submission } \\
& \text { - thorough peer review by experienced researchers in your field } \\
& \text { - rapid publication on acceptance } \\
& \text { - support for research data, including large and complex data types } \\
& \text { - gold Open Access which fosters wider collaboration and increased citations } \\
& \text { - maximum visibility for your research: over } 100 \mathrm{M} \text { website views per year }
\end{aligned}
$$

At BMC, research is always in progress.

Learn more biomedcentral.com/submissions 